\title{
Approximating surface areas by interpolations on triangulations
}

\author{
Kenta Kobayashi · Takuya Tsuchiya
}

Received: date / Accepted: date

\begin{abstract}
We consider surface area approximations by Lagrange and Crouzeix-Raviart interpolations on triangulations. For Lagrange interpolation, we give an alternative proof for Young's classical result that claims the areas of inscribed polygonal surfaces converge to the area of the original surface under the maximum angle condition on the triangulation. For Crouzeix-Raviart interpolation we show that the approximated surface areas converge to the area of the original surface without any geometric conditions on the triangulation.
\end{abstract}

Keywords Surface area, triangulations, Lagrange interpolation, Crouzeix-Raviart interpolation

Mathematics Subject Classification (2000) 65D05, 65N30, 26B15

\section{Introduction}

Let $\Omega \subset \mathbb{R}^{2}$ be a bounded domain with polygonal boundary $\partial \Omega$. For a sufficiently smooth function defined on $\Omega$, for example $f \in C^{1}(\bar{\Omega})$, the area $A(f)$ of its graph $z=f(x, y)$ is computed (and defined) by

$$
A(f)=\int_{\Omega} \sqrt{1+|\nabla f(\mathbf{x})|^{2}} \mathrm{~d} \mathbf{x} .
$$

If the smoothness assumption is weakened, however, the definition of $A(f)$ becomes rather complicated. (For the definition of surface area given by Lebesgue, see Section 2.3.) The length of a curve is defined as the limit of the length of its inscribed

Kenta Kobayashi

Graduate School of Commerce and Management,

Hitotsubashi University, Japan

E-mail: kenta.k@r.hit-u.ac.jp

Takuya Tsuchiya

Graduate School of Science and Engineering,

Ehime University, Japan

E-mail: tsuchiya@math.sci.ehime-u.ac.jp 
polygonal curves. On the contrary, the area of a surface cannot be defined as the limit of inscribed polygonal surfaces. In the 1880 s, Schwarz and Peano independently presented a well-known counter-example.
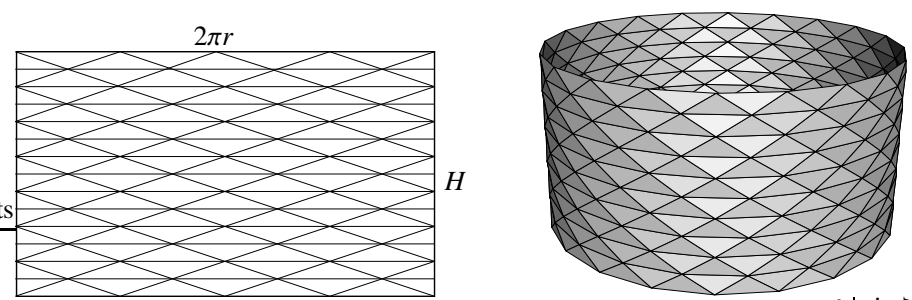

Fig. 1 Schwarz-Peano's example, called "Schwarz's lantern".

Let $\Omega$ be a rectangle of height $H$ and width $2 \pi r$. Let $m, n$ be positive integers. Suppose that this rectangle is divided into $m$ equal strips, each of height $H / m$. Each strip is then divided into isosceles triangles whose base length is $2 \pi r / n$, as depicted in Figure 1 Then, the piecewise linear map $\varphi_{\tau}: \Omega \rightarrow \mathbb{R}^{3}$ is defined by "rolling up this rectangle" so that all vertices are on the cylinder of height $H$ and radius $r$. The cylinder is then approximated by the inscribed polygonal surface, which consists of $2 m n$ congruent isosceles triangles. Because the height of each triangle is $\sqrt{(H / m)^{2}+r^{2}(1-\cos (\pi / n))^{2}}$ and the base length is $2 r \sin (\pi / n)$, the area $A_{E}$ of the inscribed polygonal surface 1 is

$$
\begin{aligned}
A_{E} & =2 m n r \sin \frac{\pi}{n} \sqrt{\left(\frac{H}{m}\right)^{2}+r^{2}\left(1-\cos \frac{\pi}{n}\right)^{2}} \\
& =2 \pi r \frac{\sin \frac{\pi}{n}}{\frac{\pi}{n}} \sqrt{H^{2}+\frac{\pi^{4} r^{2}}{4}\left(\frac{m}{n^{2}}\right)^{2}\left(\frac{\sin \frac{\pi}{2 n}}{\frac{\pi}{2 n}}\right)^{4}} .
\end{aligned}
$$

If $m, n \rightarrow \infty$, we observe

$$
\lim _{m, n \rightarrow \infty} A_{E}=2 \pi r \sqrt{H^{2}+\frac{\pi^{4} r^{2}}{4} \lim _{m, n \rightarrow \infty}\left(\frac{m}{n^{2}}\right)^{2}},
$$

and in particular,

$$
\lim _{m, n \rightarrow \infty} A_{E}=2 \pi r H \text { if and only if } \lim _{m, n \rightarrow \infty} \frac{m}{n^{2}}=0 .
$$

The example given by Schwarz and Peano has convinced mathematicians of the need to impose some geometric assumption on such triangulations to approximate the surface area by Lagrange interpolation. The known geometric conditions on triangulations are as follows: Let $\left\{\tau_{k}\right\}_{k=1}^{\infty}$ be a sequence of triangulations of $\Omega$ such

\footnotetext{
1 The sum of areas of triangles. The subscript ' $E$ ' of $A_{E}$ stands for 'Elementary'.
} 
that $\lim _{k \rightarrow \infty}\left|\tau_{k}\right|=0$, where $\left|\tau_{k}\right|:=\max _{K \in \tau_{k}} \operatorname{diam} K$. For a given continuous function $f \in C(\bar{\Omega})$, its Lagrange interpolation on the triangulation $\tau_{k}$ is denoted by $\mathcal{I}_{\tau_{k}}^{L} f$. We denote by $A_{L}(f)$ the surface area of the graph $z=f(x, y)$ in the sense of Lebesgue. We also denote by $A_{E}\left(\mathcal{I}_{\tau_{k}}^{L} f\right)$ the surface area of the Lagrange interpolation $\mathcal{I}_{\tau_{k}}^{L} f$.

Minimum angle condition. Let $\theta_{K}^{m}$ be the minimum inner angle of a triangle $K \in \tau_{k}$. Suppose that there exists a contant $\theta_{1}, 0<\theta_{1} \leq \pi / 3$, such that

$$
\theta_{1} \leq \theta_{K}^{m}, \quad \forall K \in \tau_{k}, \quad k=1,2, \cdots
$$

Then, $\left\{\tau_{k}\right\}$ is said to satisfy the minimum angle condition. Rademacher showed [15], [16] that if $\left\{\tau_{k}\right\}$ satisfies the minimum angle condition, then, for $f \in W^{1, \infty}(\Omega)$, we have

$$
\lim _{k \rightarrow \infty} A_{E}\left(\mathcal{I}_{\tau_{k}}^{L} f\right)=A_{L}(f)
$$

Maximum angle condition. Let $\theta_{K}^{M}$ be the maximum inner angle of a triangle $K \in \tau_{k}$. Suppose that there exists a constant $\theta_{2}, \pi / 3 \leq \theta_{2}<\pi$, such that

$$
\theta_{K}^{M} \leq \theta_{2}, \quad \forall K \in \tau_{k}, \quad k=1,2, \cdots
$$

Then, $\left\{\tau_{k}\right\}$ is said to satisfy the maximum angle condition. Young showed [20] that if $\left\{\tau_{k}\right\}$ satisfies the maximum angle condition, then we have (1.1) for $f \in W^{1, \infty}(\Omega)$.

Note that the minimum and maximum angle conditions were rediscovered by researchers of finite element methods some 50 years after Rademacher and Young [10]. For the above mentioned results, readers are referred to [4], [17], [18].

Recently, the authors presented the following result.

Circumradius condition. Let $R_{K}$ be the circumradius of the triangle $K \in \tau_{k}$. Suppose that

$$
\lim _{k \rightarrow \infty} \max _{K \in \tau_{k}} R_{K}=0 .
$$

Then, $\left\{\tau_{k}\right\}$ is said to satisfy the circumradius condition.

Let $R_{m, n}$ be the circumradius of the triangles in Schwarz's lantern. It has been shown in $[10]$ that

$$
\lim _{m, n \rightarrow \infty} A_{E}=2 \pi r H \text { if and only if } \lim _{m, n \rightarrow \infty} R_{m, n}=0
$$

and (1.1) was proved under the circumradius condition for $f \in W^{2,1}(\Omega)$. From these facts, we can infer that the circumradius condition is the best possible geometric condition of triangulations to assure the convergence in (1.1).

One of the aims of this paper is to give an alternate proof of Young's result using the modern theory of finite element methods. That is, in Section 3, we will show (1.1) for $f \in W^{1, \infty}(\Omega)$ under the maximum angle condition using the results given in [11].

Crouzeix-Raviart interpolation is defined using integrals of the given function on the edges of triangles. The other, more important aim of this paper is to show that 
the surface area $A_{L}(f)$ is approximated by the Crouzeix-Raviart interpolation $I_{\tau_{k}}^{C R} f$ without any geometric conditions on the triangulation. To this end, we develop the error analysis of Crouzeix-Raviart interpolation in Section 4, Using the error analysis of Crouzeix-Raviart interpolation, the main theorem (Theorem 5.1) of this paper is stated and proved in Section 5 In Section 6, we will show that the results obtained in Sections 3 and 5 for the graphs of functions on $\Omega$ hold for parametric surfaces. Finally, in Section 7 we present the results of numerical experiments to confirm the theoretical results. We also mention some concluding remarks regarding further research.

\section{Preliminaries}

2.1 Notation and the basic definitions

Let $\mathbb{R}^{d}$ be the $d$-dimensional Euclidean space. We denote the Euclidean norm of $\mathbf{x} \in$ $\mathbb{R}^{d}$ by $|\mathbf{x}|$. Let $\mathbb{R}^{d *}:=\left\{l: \mathbb{R}^{d} \rightarrow \mathbb{R}: l\right.$ is linear $\}$ be the dual space of $\mathbb{R}^{d}$. We always regard $\mathbf{x} \in \mathbb{R}^{d}$ as a column vector and $\mathbf{a} \in \mathbb{R}^{d *}$ as a row vector. For a matrix $A$ and $\mathbf{x} \in \mathbb{R}^{d}, A^{\top}$ and $\mathbf{x}^{\top}$ denote their transpositions. For a differentiable function $f$ with $d$ variables, its gradient $\nabla f=\operatorname{grad} f \in \mathbb{R}^{d *}$ is the row vector

$$
\nabla f=\nabla_{\mathbf{x}} f:=\left(\frac{\partial f}{\partial x_{1}}, \ldots, \frac{\partial f}{\partial x_{d}}\right), \quad \mathbf{x}:=\left(x_{1}, \ldots, x_{d}\right)^{\top}
$$

Let $\mathbb{N}_{0}$ be the set of nonnegative integers. For $\delta=\left(\delta_{1}, \ldots, \delta_{d}\right) \in\left(\mathbb{N}_{0}\right)^{d}$, the multiindex $\partial^{\delta}$ of partial differentiation (in the sense of distribution) is defined by

$$
\partial^{\delta}=\partial_{\mathbf{x}}^{\delta}:=\frac{\partial^{|\delta|}}{\partial x_{1}^{\delta_{1}} \ldots \partial x_{d}^{\delta_{d}}}, \quad|\delta|:=\delta_{1}+\ldots+\delta_{d}
$$

If $d=2$, we use the notation $f_{x}$ and $f_{y}$ instead of $\partial f / \partial x$ and $\partial f / \partial y$, respectively.

Let $\Omega \subset \mathbb{R}^{2}$ be a (bounded) domain. The usual Lebesgue space is denoted by $L^{p}(\Omega)$ for $1 \leq p \leq \infty$. For a positive integer $k$, the Sobolev space $W^{k, p}(\Omega)$ is defined by $W^{k, p}(\Omega):=\left\{v \in L^{p}(\Omega)\left|\partial^{\delta} v \in L^{p}(\Omega),\right| \delta \mid \leq k\right\}$. The norm and semi-norm of $W^{k, p}(\Omega)$ are defined, for $1 \leq p<\infty$, by

$$
|v|_{k, p, \Omega}:=\left(\sum_{|\delta|=k}\left|\partial^{\delta} v\right|_{0, p, \Omega}^{p}\right)^{1 / p}, \quad\|v\|_{k, p, \Omega}:=\left(\sum_{0 \leq m \leq k}|v|_{m, p, \Omega}^{p}\right)^{1 / p}
$$

and $|v|_{k, \infty, \Omega}:=\max _{|\delta|=k}\left\{\underset{\mathbf{x} \in \Omega}{\operatorname{ess} \sup }\left|\partial^{\delta} v(\mathbf{x})\right|\right\},\|v\|_{k, \infty, \Omega}:=\max _{0 \leq m \leq k}\left\{|v|_{m, \infty, \Omega}\right\}$.

Let $\mathbf{f}: \Omega \rightarrow \mathbb{R}^{d}$ with $\mathbf{f}=\left(f^{1}, \cdots, f^{d}\right)$. If $f^{i} \in W^{k, p}(\Omega), i=1, \cdots, d$, we write $\mathbf{f}$ as $\mathbf{f} \in W^{k, p}\left(\Omega ; \mathbb{R}^{d}\right)$. Their norms are defined similarly. 
2.2 Triangulation of bounded polygonal domains and Lagrange and Crouzeix-Raviart interpolations

Throughout this paper, $K$ is a triangle in $\mathbb{R}^{2}$. Let $\Omega \subset \mathbb{R}^{2}$ be a bounded polygonal domain. A triangulation $\tau$ of $\Omega$ is a set of triangles that satisfies the following properties.

$-\bar{\Omega}=\bigcup_{K \in \tau} K$.

- If $K_{1}, K_{2} \in \tau$, we have either $K_{1} \cap K_{2}=\emptyset$, or $K_{1} \cap K_{2}$ is a vertex or an edge of both $K_{1}$ and $K_{2}$.

Because of the second property, the triangulations discussed here are sometimes called face-to-face triangulations. For a triangulation $\tau$, the fineness $|\tau|$ is defined by

$$
|\tau|:=\max _{K \in \tau} \operatorname{diam} K
$$

We denote by $\mathcal{P}_{1}$ the set of all polynomials with two variables whose orders are at most 1 . For a triangulation $\tau$ of $\Omega$, we define the set $S_{\tau}$ of all piecewise linear continuous functions by

$$
S_{\tau}:=\left\{f \in C^{0}(\bar{\Omega})|f|_{K} \in \mathcal{P}_{1}, \forall K \in \tau\right\} .
$$

Let $\mathbf{x}_{i}, i=1,2,3$ be vertices of a triangle $K$. Let $e_{i}$ be the edge of $K$ opposite to $\mathbf{x}_{i}$. For a continuous function $f \in C(K)$, the Lagrange interpolation $\mathcal{I}_{K}^{L} f \in \mathcal{P}_{1}$ on $K$ is defined by $f\left(\mathbf{x}_{i}\right)=\left(\mathcal{I}_{K}^{L} f\right)\left(\mathbf{x}_{i}\right), i=1,2,3$. It is clear that, for $f \in C(\bar{\Omega})$ and a triangulation $\tau$ of $\Omega$, we can define the Lagrange interpolation $\mathcal{I}_{\tau}^{L} f \in S_{\tau}$ as

$$
\left.\mathcal{I}_{\tau}^{L} f\right|_{K}=\mathcal{I}_{K}^{L} f, \quad \forall K \in \tau .
$$

Next, let the polynomial $\theta_{i} \in \mathcal{P}_{1}, i=1,2,3$ be defined by

$$
\int_{e_{i}} \theta_{i}(\mathbf{x}) \mathrm{d} s=1, \quad \int_{e_{i}} \theta_{j}(\mathbf{x}) \mathrm{d} s=0, \quad i \neq j .
$$

Using the barycentric coordinate $\lambda_{i}(\mathbf{x})$ on $K$, this can be written as

$$
\theta_{i}(\mathbf{x}):=\frac{1}{\left|e_{i}\right|}\left(1-2 \lambda_{i}(\mathbf{x})\right)
$$

For a function $v \in W^{1,1}(K)$ on $K$, the (non-conforming) Crouzeix-Raviart interpolation $\mathcal{I}_{K}^{C R} v$ is defined by

$$
\mathcal{I}_{K}^{C R} v:=\sum_{i=1}^{3}\left(\int_{e_{i}} v \mathrm{~d} s\right) \theta_{i} .
$$

Note that $\mathcal{I}_{K}^{C R} v$ is well-defined because the trace operator $\gamma_{i}: W^{1,1}(K) \rightarrow L^{1}\left(e_{i}\right)$ is continuous. Moreover, we have

$$
\int_{e_{i}}\left(v-\mathcal{I}_{K}^{C R} v\right) \mathrm{d} s=0, \quad i=1,2,3 .
$$


The Crouzeix-Raviart interpolation $\mathcal{I}_{K}^{C R} v \in \mathcal{P}_{1}$ may be defined using this equality. The global (non-conforming) Crouzeix-Raviart interpolation $\mathcal{I}_{\tau}^{C R} f \in L^{\infty}(\Omega)$ on $\tau$ is defined by

$$
\left.\mathcal{I}_{\tau}^{C R} f\right|_{K}=\mathcal{I}_{K}^{C R} f, \quad \forall K \in \tau
$$

Note that $\mathcal{I}_{\tau}^{C R} f$ is not continuous in general. Let $K_{1}, K_{2} \in \tau$ be two adjacent triangles in $\tau$. Then, on $e=K_{1} \cap K_{2}, \mathcal{I}_{\tau}^{C R} f$ is continuous only at the midpoint of $e$. In Figure 2 we show the graphs of Lagrange and Crouzeix-Raviart interpolations of the function $f(x, y)=\sqrt{a^{2}-x^{2}}, a=1.1$ on a triangulation on $\Omega:=(-1,1) \times(-1,1)$, similar to the one depicted in Figure 1
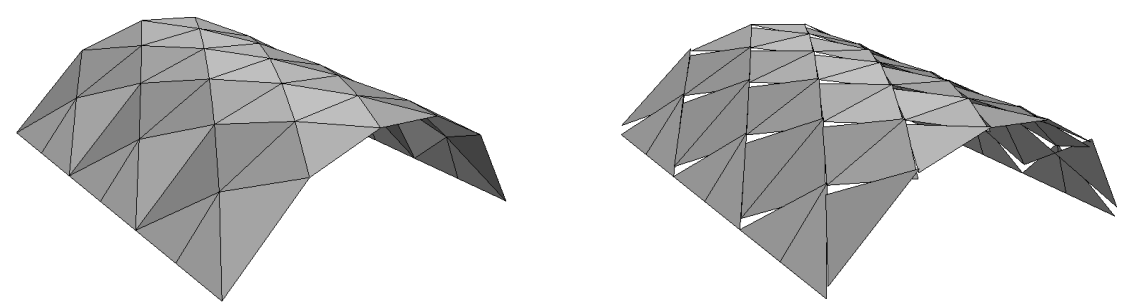

Fig. 2 Lagrange and Crouzeix-Raviart interpolations.

For the definitions of Lagrange and Crouzeix-Raviart interpolations, readers are referred to textbooks on finite element methods, such as [2], [5], and [6].

2.3 Lebesgue's definition of the surface area and Tonelli's theorem

At present, the most general definition of surface area is that of Lebesgue. Let $\Omega:=$ $(a, b) \times(c, d) \subset \mathbb{R}^{2}$ be a rectangle and $\tau_{n}$ be a sequence of triangulations of $\Omega$ such that $\lim _{n \rightarrow \infty}\left|\tau_{n}\right|=0$. Let $f \in C^{0}(\bar{\Omega})$ be a given continuous function. Let $f_{n} \in S_{\tau_{n}}$ be such that $\left\{f_{n}\right\}_{n=1}^{\infty}$ converges uniformly to $f$ on $\bar{\Omega}$. Note that the graph of $z=f_{n}(x, y)$ is a set of triangles, and its area is defined as a sum of these triangular areas. We denote this area by $A_{E}\left(f_{n}\right)$, and have

$$
A_{E}\left(f_{n}\right)=\int_{\Omega} \sqrt{1+\left|\nabla f_{n}\right|^{2}} \mathrm{~d} x
$$

Let $\Phi_{f}$ be the set of all such sequences $\left\{\left(f_{n}, \tau_{n}\right)\right\}_{n=1}^{\infty}$. Then, the area $A_{L}(f)=A_{L}(f ; \Omega)$ of the graph $z=f(x, y)$ is defined by

$$
A_{L}(f)=A_{L}(f ; \Omega):=\inf _{\left\{\left(f_{n}, \tau_{n}\right)\right\} \in \Phi_{f}} \liminf _{n \rightarrow \infty} A_{E}\left(f_{n}\right) .
$$

This $A_{L}(f)$ is called the surface area of $z=f(x, y)$ in the Lebesgue sense. For a fixed $f, A_{L}(f ; \Omega)$ is additive and continuous with respect to the domain $\Omega$. Tonelli presented the following theorem. 
For a continuous function $f \in C^{0}(\bar{\Omega})$, we define $W_{1}(x), W_{2}(y)$ by

$$
\begin{aligned}
& W_{1}(x):=\sup _{\tau(y)} \sum_{i}\left|f\left(x, y_{i-1}\right)-f\left(x, y_{i}\right)\right|, \quad x \in(a, b), \\
& W_{2}(y):=\sup _{\tau(x)} \sum_{j}\left|f\left(x_{j-1}, y\right)-f\left(x_{j}, y\right)\right|, \quad y \in(c, d),
\end{aligned}
$$

where $\tau(y), \tau(x)$ are the subdivisions $c=y_{0}<y_{1}<\cdots<y_{N}=d$ and $a=x_{0}<$ $x_{1}<\cdots<x_{M}=b$, respectively, and 'sup' is taken for all such subdivisions. Then, a function $f$ has bounded variation in the Tonelli sense if

$$
\int_{a}^{b} W_{1}(x) \mathrm{d} x+\int_{c}^{d} W_{2}(y) \mathrm{d} y<\infty .
$$

Additionally, a function $f$ is said to be absolutely continuous in the Tonelli sense if, for almost all $y \in(c, d)$ and $x \in(a, b)$, the functions $g(x):=f(x, y)$ and $h(y):=f(x, y)$ are absolutely continuous on $(a, b)$ and $(c, d)$, respectively. The following theorem is well-known.

Theorem 2.1 (Tonelli) For a continuous function $f \in C(\bar{\Omega})$ defined on a rectangular domain $\Omega$, its graph $z=f(x, y)$ has finite area $A_{L}(f)<\infty$ if and only if $f$ has bounded variation in the Tonelli sense. If this is the case, we have

$$
A_{L}(f) \geq \int_{\Omega} \sqrt{1+|\nabla f(\mathbf{x})|^{2}} \mathrm{~d} \mathbf{x}
$$

In the above inequality, the equality holds if and only if $f$ is absolutely continuous in the Tonelli sense.

For a proof of this theorem, see [19, Chapter V, pp.163-185]. It follows from Tonelli's theorem that if $f \in W^{1, \infty}(\Omega)$, then the area $A_{L}(f)$ is finite and the equality holds in (2.1).

\subsection{Affine linear transformation of triangles}

Let $\widehat{K}$ be the reference triangle with vertices $\hat{\mathbf{x}}_{1}=(0,0)^{\top}, \hat{\mathbf{x}}_{2}=(1,0)^{\top}$, and $\hat{\mathbf{x}}_{3}=$ $(0,1)^{\top}$. For $\alpha, 0<\alpha \leq 1$, let $K_{\alpha}$ and $\widetilde{K}_{\alpha}$ be the triangles with vertices $(0,0)^{\top},(1,0)^{\top}$, $(0, \alpha)^{\top}$, and $\mathbf{x}_{1}=(0,0)^{\top}, \mathbf{x}_{2}=(1,0)^{\top}, \mathbf{x}_{3}=(\alpha s, \alpha t)^{\top}$, respectively, where $s^{2}+t^{2}=1$, $t>0$. Without loss of generality, we may assume that $e_{1}$ is the longest edge of $\widetilde{K}_{\alpha}$. Let $\theta$ be the angle between $e_{2}$ and $e_{3}$ in $\widetilde{K}_{\alpha}$. Then, $s=\cos \theta, t=\sin \theta$, and the assumption that $e_{1}$ is the longest yields

$$
s=\cos \theta \leq \frac{\alpha}{2} \leq \frac{1}{2}, \quad \frac{\pi}{3} \leq \theta<\pi .
$$

Note that an arbitrary triangle in $\mathbb{R}^{2}$ can be transformed to $\widetilde{K}_{\alpha}$ by a sequence of scaling, translation, rotation, and mirror imaging. 


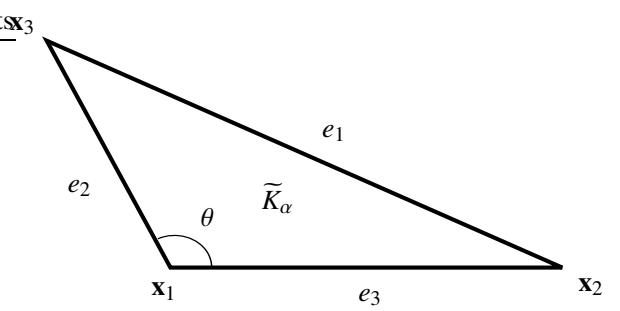

Fig. 3 The triangle under consideration. The vertices are $\mathbf{x}_{1}=(0,0)^{\top}, \mathbf{x}_{2}=(1,0)^{\top}$, and $\mathbf{x}_{3}=(\alpha s, \alpha t)^{\top}$, where $s^{2}+t^{2}=1, t>0$, and $0<\alpha \leq 1$. We assume that $\alpha=\left|e_{2}\right| \leq\left|e_{3}\right|=1 \leq\left|e_{1}\right|$.

We define the $2 \times 2$ matrices as

$$
A:=\left(\begin{array}{ll}
1 & s \\
0 & t
\end{array}\right), \quad B:=A^{-1}=\left(\begin{array}{cc}
1 & -s t^{-1} \\
0 & t^{-1}
\end{array}\right) .
$$

Then, $K_{\alpha}$ can be transformed to $\widetilde{K}_{\alpha}$ by the transformation $\mathbf{y}=A \mathbf{x}$. Moreover, a function $v(\mathbf{y}) \in W^{1, p}\left(\widetilde{K}_{\alpha}\right)$ is pulled-back to the function $\hat{v}(\mathbf{x}) \in W^{1, p}\left(K_{\alpha}\right)$ as $\hat{v}(\mathbf{x}):=$ $v(A \mathbf{x})=v(\mathbf{y})$. Then, we have $\nabla_{\mathbf{x}} \hat{v}=\left(\nabla_{\mathbf{y}} v\right) A, \nabla_{\mathbf{y}} v=\left(\nabla_{\mathbf{x}} \hat{v}\right) B$, and $\left|\nabla_{\mathbf{y}} v\right|^{2}=\left|\left(\nabla_{\mathbf{x}} \hat{v}\right) B\right|^{2}=$ $\left(\nabla_{\mathbf{x}} \hat{v}\right) B B^{\top}\left(\nabla_{\mathbf{x}} \hat{v}\right)^{\top}$. A simple computation yields that $A^{\top} A$ has eigenvalues $1 \pm|s|$, and $B B^{\top}$ has eigenvalues $1 /(1 \pm|s|)=(1 \mp|s|) / t^{2}$. Hence, we have

$$
\frac{1-|s|}{t^{2}}\left|\nabla_{\mathbf{x}} \hat{v}\right|^{2} \leq\left|\nabla_{\mathbf{y}} v\right|^{2} \leq \frac{1+|s|}{t^{2}}\left|\nabla_{\mathbf{x}} \hat{v}\right|^{2} .
$$

Note that, for $N$ positive real numbers $U_{1}, \ldots, U_{N}$, the following inequalities hold:

$$
\begin{aligned}
& \sum_{k=1}^{N} U_{k}^{p} \leq N^{\tau(p)}\left(\sum_{k=1}^{N} U_{k}^{2}\right)^{p / 2}, \quad \tau(p):=\left\{\begin{array}{ll}
1-p / 2, & 1 \leq p \leq 2 \\
0, & 2 \leq p<\infty
\end{array},\right. \\
& \left(\sum_{k=1}^{N} U_{k}^{2}\right)^{p / 2} \leq N^{\gamma(p)} \sum_{k=1}^{N} U_{k}^{p}, \quad \gamma(p):=\left\{\begin{array}{ll}
0, & 1 \leq p \leq 2 \\
p / 2-1, & 2 \leq p<\infty
\end{array} .\right.
\end{aligned}
$$

Combining (2.3) with (2.4), 2.5), and noting that the determinant of $A$ is $t$, we have, for $1 \leq p<\infty$,

$$
\begin{aligned}
|v|_{1, p, \widetilde{K}_{\alpha}}^{p} & =\int_{\widetilde{K}_{\alpha}} \sum_{|\delta|=1}\left|\partial_{\mathbf{y}}^{\delta} v(\mathbf{y})\right|^{p} \mathrm{~d} \mathbf{y} \geq 2^{-\gamma(p)} \int_{\widetilde{K}_{\alpha}}\left(\left|\nabla_{\mathbf{y}} v(\mathbf{y})\right|^{2}\right)^{p / 2} \mathrm{~d} \mathbf{y} \\
& \geq 2^{-\gamma(p)}\left(\frac{1-|s|}{t^{2}}\right)^{p / 2} \int_{\widetilde{K}_{\alpha}}\left(\left|\nabla_{\mathbf{x}} \hat{v}(\mathbf{x})\right|^{2}\right)^{p / 2} \mathrm{~d} \mathbf{y} \\
& =2^{-\gamma(p)}\left(\frac{1-|s|}{t^{2}}\right)^{p / 2} t \int_{K_{\alpha}}\left(\left|\nabla_{\mathbf{x}} \hat{v}(\mathbf{x})\right|^{2}\right)^{p / 2} \mathrm{~d} \mathbf{x} \\
& \geq 2^{-(\tau(p)+\gamma(p))}\left(\frac{1-|s|}{t^{2}}\right)^{p / 2} t \int_{K_{\alpha}} \sum_{|\delta|=1}\left|\partial_{\mathbf{x}}^{\delta} \hat{v}(\mathbf{x})\right|^{p} \mathrm{~d} \mathbf{x}
\end{aligned}
$$




$$
=2^{-(\tau(p)+\gamma(p))}\left(\frac{1-|s|}{t^{2}}\right)^{p / 2} t|\hat{v}|_{1, p, K_{\alpha}}^{p}
$$

and similarly,

$$
|v|_{1, p, \widetilde{K}_{\alpha}}^{p} \leq 2^{\tau(p)+\gamma(p)}\left(\frac{1+|s|}{t^{2}}\right)^{p / 2} t \mid \hat{v}_{1, p, K_{\alpha}}^{p} .
$$

Let $K$ be an arbitrary triangle and $K_{1}$ be the right triangle obtained by a composition of parallel translation, mirror imaging, and $A^{-1}$. As before, any $v \in W^{1, p}(K)$ may be pulled-back to the function $\hat{v}:=v \circ \rho \in W^{1, p}\left(K_{1}\right)$. Then, in exactly the same manner, we obtain

$$
2^{-\eta(p)} \frac{(1-|s|)^{1 / 2}}{t^{1-1 / p}}|\hat{v}|_{1, p, K_{1}} \leq|v|_{1, p, K} \leq 2^{\eta(p)} \frac{(1+|s|)^{1 / 2}}{t^{1-1 / p}}|\hat{v}|_{1, p, K_{1}},
$$

where $\eta(p):=1 / p-1 / 2$ for $1 \leq p \leq 2$ and $\eta(p):=1 / 2-1 / p$ for $2 \leq p<\infty$. By letting $p \rightarrow \infty$, we also obtain

$$
\frac{(1-|s|)^{1 / 2}}{\sqrt{2} t}|\hat{v}|_{1, \infty, K_{1}} \leq|v|_{1, \infty, K} \leq \frac{\sqrt{2}(1+|s|)^{1 / 2}}{t}|\hat{v}|_{1, \infty, K_{1}} .
$$

\section{Approximating the surface area by Lagrange interpolation}

Let $K_{1}$ be a right triangle whose vertices are $\hat{\mathbf{x}}_{1}:=(0,0)^{\top}, \hat{\mathbf{x}}_{2}:=\left(h_{1}, 0\right)^{\top}$, and $\hat{\mathbf{x}}_{3}:=$ $\left(0, h_{2}\right)^{\top}$, where $0<h_{2} \leq h_{1}$. Let $K$ be the triangle whose vertices are defined by $\mathbf{x}_{i}:=$ $A \hat{\mathbf{x}}_{i}, i=1,2,3$, where the matrix $A$ is defined by (2.2). Without loss of generality, we may assume that the angle at the vertex $A \mathbf{x}_{1}$ is the maximum angle of $K$. Note that an arbitrary triangle is obtained from $K$ by a combination of rotation, translation, and mirror imaging.

As before, an arbitrary function $\hat{v} \in W^{1, \infty}\left(K_{1}\right)$ is pulled-back to $v(\mathbf{x}):=\hat{v}\left(A^{-1} \mathbf{x}\right)$. Then, their Lagrange interpolations $\mathcal{I}_{K_{1}}^{L} \hat{v}$ and $\mathcal{I}_{K}^{L} v$ are defined as

$$
\begin{aligned}
& \left(I_{K_{1}}^{L} \hat{v}\right)(\widehat{X}, \widehat{Y})=\widehat{P X}+\widehat{R Y}+\widehat{R}, \quad \widehat{P}:=\frac{\hat{v}\left(\hat{\mathbf{x}}_{2}\right)-\hat{v}\left(\hat{\mathbf{x}}_{1}\right)}{\left|\hat{\mathbf{x}}_{2}-\hat{\mathbf{x}}_{1}\right|}, \widehat{Q}:=\frac{\hat{v}\left(\hat{\mathbf{x}}_{3}\right)-\hat{v}\left(\hat{\mathbf{x}}_{1}\right)}{\left|\hat{\mathbf{x}}_{3}-\hat{\mathbf{x}}_{1}\right|} \\
& \left(\mathcal{I}_{K}^{L} v\right)(X, Y)=P X+Q Y+R, \quad P=\widehat{P}, \quad Q=-\frac{s}{t} \widehat{P}+\frac{1}{t} \widehat{Q}
\end{aligned}
$$

Therefore, we see that

$$
\left|\mathcal{I}_{K_{1}}^{L} \hat{v}\right|_{1, \infty, K_{1}} \leq|\hat{v}|_{1, \infty, K_{1}}, \quad\left|\mathcal{I}_{K}^{L} v\right|_{1, \infty, K} \leq \frac{1+|s|}{t}\left|\mathcal{I}_{K_{1}}^{L} \hat{v}\right|_{1, \infty, K_{1}}
$$

Combining these inequalities with (2.6), we have

$$
\left|\mathcal{I}_{K}^{L} v\right|_{1, \infty, K} \leq \frac{4}{\sin \theta_{K}}|v|_{1, \infty, K}
$$

where $\theta_{K}$ is the maximum angle of $K$. Note that, in general, the Sobolev norm is affected by a rotation. Hence, we have shown the following lemma. 
Lemma 3.1 Let $\Omega \subset \mathbb{R}^{2}$ be a bounded polygonal domain and $\tau$ be a triangulation of $\Omega$. Suppose that $\tau$ satisfies the maximum angle condition, that is, there exists $\theta_{2}$, $\pi / 3 \leq \theta_{2}<\pi$, such that $\theta_{K} \leq \theta_{2}$ for any $K \in \tau$. Then, there exists a constant $C_{1}$ depending only on $\theta_{1}$ such that

$$
\left\|\mathcal{I}_{\tau}^{L}\right\|_{\mathcal{L}\left(W^{1, \infty}(\Omega), W^{1, \infty}(\Omega)\right)} \leq C_{1},
$$

where $\left\|\mathcal{I}_{\tau}^{L}\right\|_{\mathcal{L}\left(W^{1, \infty}(\Omega), W^{1, \infty}(\Omega)\right)}$ is the operator norm of $\mathcal{I}_{\tau}^{L}: W^{1, \infty}(\Omega) \rightarrow W^{1, \infty}(\Omega)$.

Lemma 3.1 provides an alternate proof of the following classical result.

Theorem 3.2 (Young [20]) Let $\Omega \subset \mathbb{R}^{2}$ be a bounded domain and $\left\{\tau_{k}\right\}_{k=1}^{\infty}$ be a sequence of triangulations of $\Omega$ with $\lim _{k \rightarrow \infty}\left|\tau_{k}\right|=0$ that satisfies the maximum angle condition. That is, there exists $\theta_{2}, \pi / 3 \leq \theta_{2}<\pi$, such that $\theta_{K} \leq \theta_{2}$ for any $K \in \tau_{k}$, $k=1,2, \cdots$. Then, for any $f \in W^{1, \infty}(\Omega)$, we have

$$
\lim _{k \rightarrow \infty} A_{E}\left(\mathcal{I}_{\tau_{k}}^{L} f\right)=A_{L}(f)
$$

Proof First, we note that, for $f, g \in W^{1,1}(K)$,

$$
\left|\int_{K} \sqrt{1+|\nabla f|^{2}} \mathrm{~d} \mathbf{x}-\int_{K} \sqrt{1+|\nabla g|^{2}} \mathrm{~d} \mathbf{x}\right| \leq|f-g|_{1,1, K},
$$

because

$$
\frac{\left|\partial^{\gamma} f+\partial^{\gamma} g\right|}{\sqrt{1+|\nabla f|^{2}}+\sqrt{1+|\nabla g|^{2}}} \leq 1, \quad \gamma=(1,0),(0,1) .
$$

Let $\varepsilon>0$ be arbitrarily taken and fixed. We may take $f_{\varepsilon} \in W^{2, \infty}(\Omega)$ such that $\mid f-$ $\left.f_{\varepsilon}\right|_{1, \infty, \Omega}<\varepsilon$. Recall that we have the estimation

$$
\left|f_{\varepsilon}-\mathcal{I}_{\tau_{k}}^{L} f_{\varepsilon}\right|_{1, \infty, \Omega} \leq C_{2} R_{k}\left|f_{\varepsilon}\right|_{2, \infty, \Omega},
$$

where $R_{k}:=\max _{K \in \tau_{k}} R_{K}$ and $C_{2}$ is a constant that is independent of $\tau_{k}$ and $f_{\varepsilon}$ [9], [10], [11]. If the sequence of triangulations $\left\{\tau_{k}\right\}$ satisfies the maximum angle condition, then it satisfies the circumradius condition. Hence, we have $\lim _{k \rightarrow \infty} R_{k}=0$.

There exists an integer $N$ such that, for any integer $k \geq N$, we have $C_{2} R_{k}\left|f_{\varepsilon}\right|_{2, \infty, \Omega}<$ $\varepsilon$. Let $|\Omega|$ be the area of $\Omega$. It follows from Lemma 3.1 and (3.2) that, for $k \geq N$,

$$
\begin{aligned}
\mid A_{L}(f)- & A_{E}\left(\mathcal{I}_{\tau_{k}}^{L} f\right)|\leq| f-\left.\mathcal{I}_{\tau_{k}}^{L} f\right|_{1,1, \Omega} \\
& \leq\left|f-f_{\varepsilon}\right|_{1,1, \Omega}+\left|f_{\varepsilon}-\mathcal{I}_{\tau_{k}}^{L} f_{\varepsilon}\right|_{1,1, \Omega}+\left|\mathcal{I}_{\tau_{k}}^{L}\left(f_{\varepsilon}-f\right)\right|_{1,1, \Omega} \\
& \leq|\Omega|\left(\left|f-f_{\varepsilon}\right|_{1, \infty, \Omega}+\left|f_{\varepsilon}-\mathcal{I}_{\tau_{k}}^{L} f_{\varepsilon}\right|_{1, \infty, \Omega}+\left|\mathcal{I}_{\tau_{k}}^{L}\left(f_{\varepsilon}-f\right)\right|_{1, \infty, \Omega}\right) \\
& \leq|\Omega|\left(\varepsilon+C_{2} R_{k}\left|f_{\varepsilon}\right|_{2, \infty, \Omega}+\left|\mathcal{I}_{\tau_{k}}^{L}\left(f_{\varepsilon}-f\right)\right|_{1, \infty, \Omega}\right) \\
& \leq|\Omega|\left(2 \varepsilon+|| \mathcal{I}_{\tau_{k}}^{L} \|_{\mathcal{L}\left(W^{1, \infty}(\Omega), W^{1, \infty}(\Omega)\right)}\left|f-f_{\varepsilon}\right|_{1, \infty, \Omega}\right) \\
& <|\Omega|\left(2+C_{1}\right) \varepsilon .
\end{aligned}
$$

Because $\varepsilon$ is arbitrary, these inequalities indicate that (3.1) holds. 
Remark: Here, we describe Young's original proof of Theorem 3.2 concisely. Let $R:=$ $(a, c) \times(b, d)$ be a rectangle. Let $x(u, v)$ and $y(u, v)$ be sufficiently smooth functions defined on $(u, v) \in R$, and $B(u, v)$ be defined by

$$
B(u, v):=\frac{\partial x}{\partial u} \frac{\partial y}{\partial v}-\frac{\partial x}{\partial v} \frac{\partial y}{\partial u}
$$

The rectangle $R$ is divided into small rectangles with segments that are parallel to $u$ and $v$-axes. As a result, $R$ is divided into small (possibly very thin) sub-rectangles. Furthermore, each sub-rectangle is divided into two semi-rectangles (triangles) by means of the diagonal, sloping down from left to right.

Let $h, k$ be sufficiently small reals such that $h k>0$, and $(u, v)^{\top},(u+h, v)^{\top}$, $(u+h, v+k)^{\top},(u, v+k)^{\top}$ be the corner points of a sub-rectangle. Define

$$
\begin{aligned}
\left|D_{n}\right|:=\frac{1}{2} \mid(x(u+h, v) & -x(u, v))(y(u, v+k)-y(u, v)) \\
& -(y(u+h, v)-y(u, v))(x(u, v+k)-x(u, v)) \mid
\end{aligned}
$$

for one triangle, and also a similar expression for the other triangle. Young considered $\sum_{n}\left|D_{n}\right|$, where the summation is taken for all such triangles. He proved that

$$
\lim _{\bar{h}, \bar{k} \rightarrow 0} \sum_{n}\left|D_{n}\right|=\int_{a}^{b} \int_{c}^{d}|B(u, v)| d u d v, \quad \bar{h}:=\max h, \quad \bar{k}:=\max k
$$

by rather measure theoretic manner (considering Stieltjes integrals). The conclusion was immediately extended to the case of surface areas. Then, he "skewed" triangles in sub-rectangles so that one of angles of every triangle in the $(u, v)$-plane lies between $0<\gamma$ and $\pi-\gamma$, and he finally claimed that Theorem 3.2 is valid.

Therefore, the strategy of his proof was "compress right triangles perpendicularly and skew them", and is similar to ours.

\section{Error analysis of Crouzeix-Raviart interpolation}

Let $\gamma \in \mathbb{N}_{0}^{2}$ be a multi-index with $|\gamma|=1$. The sets $\Xi_{p}^{\gamma} \subset W^{1, p}(\widehat{K}), 1 \leq p \leq \infty$, are defined by

$$
\begin{aligned}
& \Xi_{p}^{(1,0)}:=\left\{v \in W^{1, p}(\widehat{K}) \mid \int_{0}^{1} v(s, 0) \mathrm{d} s=0\right\}, \\
& \Xi_{p}^{(0,1)}:=\left\{v \in W^{1, p}(\widehat{K}) \mid \int_{0}^{1} v(0, s) \mathrm{d} s=0\right\} .
\end{aligned}
$$

Similarly, for an arbitrary triangle $K \subset \mathbb{R}^{2}, \mathcal{E}_{p}(K), \Phi_{p}(K) \subset W^{1, p}(K)$ are defined by

$$
\begin{aligned}
& \Phi_{p}(K):=\left\{v \in W^{1, p}(K) \mid \int_{K} v \mathrm{~d} \mathbf{x}=0\right\}, \\
& \mathcal{E}_{p}(K):=\left\{v \in W^{1, p}(K) \mid \int_{e_{i}} v \mathrm{~d} s=0, i=1,2,3\right\} .
\end{aligned}
$$


From the definition, it is clear that $\mathcal{E}_{p}(\widehat{K}) \subset \Xi_{P}^{\gamma},|\gamma|=1$. Then, the constant $A_{p}$ and $B_{p}(K)$ are defined for $p \in[1, \infty]$ by

$$
\begin{gathered}
A_{p}:=\sup _{v \in \Xi_{p}^{(1,0)}} \frac{|v|_{0, p, \widehat{K}}}{|v|_{1, p, \widehat{K}}}=\sup _{v \in \Xi_{p}^{(0,1)}} \frac{|v|_{0, p, \widehat{K}}}{|v|_{1, p, \widehat{K}}}, \\
B_{p}(K):=\sup _{v \in \Phi_{p}(K)} \frac{|v|_{0, p, K}}{|v|_{1, p, K}}, \quad C_{p}(K):=\sup _{v \in \mathcal{E}_{p}(K)} \frac{|v|_{0, p, K}}{|v|_{1, p, K}} .
\end{gathered}
$$

The constant $A_{p}$ is called the Babuška-Aziz constant for $p, 1 \leq p \leq \infty$. According to Liu-Kikuchi [13], $A_{2}$ is the maximum positive solution of the equation $1 / x+$ $\tan (1 / x)=0$, and $A_{2} \approx 0.49291$. Babuška-Aziz [1] and Kobayashi-Tsuchiya [9] showed the following lemma.

Lemma 4.1 We have $A_{p}<\infty, 1 \leq p \leq \infty$.

Similarly, the following lemma holds.

Lemma 4.2 We have $B_{p}(\widehat{K})<\infty, 1 \leq p \leq \infty$.

Proof The proof is by contradiction. Suppose that $B_{p}(\widehat{K})=\infty$. Then, there exists $\left\{w_{k}\right\}_{k=1}^{\infty} \subset \Phi_{p}(\widehat{K})$ such that

$$
\left|w_{k}\right|_{0, p, \widehat{K}}=1, \quad \lim _{k \rightarrow \infty}\left|w_{k}\right|_{1, p, \widehat{K}}=0 .
$$

By [5, Theorem 3.1.1], there is a constant $C(\widehat{K}, p)$ such that

$$
\inf _{q \in \mathbb{R}}\|v+q\|_{1, p, \widehat{K}} \leq C(\widehat{K}, p)|v|_{1, p, \widehat{K}}, \quad \forall v \in W^{1, p}(\widehat{K}) .
$$

Therefore, there exists $\left\{q_{k}\right\} \subset \mathbb{R}$ such that

$$
\begin{gathered}
\inf _{q \in \mathbb{R}}\left\|w_{k}+q\right\|_{1, p, \widehat{K}} \leq\left\|w_{k}+q_{k}\right\|_{1, p, \widehat{K}} \leq \inf _{q \in \mathbb{R}}\left\|w_{k}+q\right\|_{1, p, \widehat{K}}+\frac{1}{k}, \\
\lim _{k \rightarrow \infty}\left\|w_{k}+q_{k}\right\|_{1, p, \widehat{K}} \leq \lim _{k \rightarrow \infty}\left(C(\widehat{K}, p)\left|w_{k}\right|_{1, p, \widehat{K}}+\frac{1}{k}\right)=0 .
\end{gathered}
$$

As the sequence $\left\{w_{k}\right\} \subset W^{1, p}(\widehat{K})$ is bounded, $\left\{q_{k}\right\} \subset \mathbb{R}$ is also bounded. Thus, there exists a subsequence $\left\{q_{k_{i}}\right\}$ such that $q_{k_{i}}$ converges to $\bar{q} \in \mathbb{R}$. In particular, we have

$$
\lim _{k_{i} \rightarrow \infty}\left\|w_{k_{i}}+\bar{q}\right\|_{1, p, \widehat{K}}=0
$$

Hence, we have

$$
0=\lim _{k \rightarrow \infty} \int_{\widehat{K}}\left(w_{k_{i}}+\bar{q}\right) \mathrm{d} \mathbf{x}=\int_{\widehat{K}} \bar{q} \mathrm{~d} \mathbf{x},
$$

because $w_{k_{i}} \in \Phi_{p}(\widehat{K})$. Hence, we conclude that $\bar{q}=0$ and $\lim _{k_{i} \rightarrow \infty}\left\|w_{k_{i}}\right\|_{1, p, \widehat{K}}=0$, which contradicts $\lim _{k_{i} \rightarrow \infty}\left|w_{k_{i}}\right|_{0, p, \widehat{K}}=1$. 
Let $\alpha \in(0,1]$ and $F_{\alpha}: \mathbb{R}^{2} \rightarrow \mathbb{R}^{2}$ be defined by $F_{\alpha}(x, y):=(x, \alpha y)^{\top},(x, y)^{\top} \in$ $\mathbb{R}^{2}$. An arbitrary $v \in W^{1, p}\left(K_{\alpha}\right)$ is pulled-back to $\hat{v}:=v \circ F_{\alpha} \in W^{1, p}(\widehat{K})$. From the definitions, it is clear that if $v \in \mathcal{E}_{p}\left(K_{\alpha}\right)$ or $v \in \Phi_{p}\left(K_{\alpha}\right)$, then $\hat{v} \in \mathcal{E}_{p}(\widehat{K})$ or $\hat{v} \in \Phi_{p}(\widehat{K})$, respectively. Because

$$
|v|_{0, p, K_{\alpha}}^{p}=\alpha|\hat{v}|_{0, p, \widehat{K}}^{p}, \quad\left|v_{x}\right|_{0, p, K_{\alpha}}^{p}=\alpha\left|\hat{v}_{x}\right|_{0, p, \widehat{K}}^{p}, \quad\left|v_{y}\right|_{0, p, K_{\alpha}}^{p}=\frac{1}{\alpha^{p-1}}\left|\hat{v}_{y}\right|_{0, p, \widehat{K}}^{p}
$$

we have, for $v \in W^{1, p}\left(K_{\alpha}\right)$,

$$
\frac{|v|_{0, p, K_{\alpha}}^{p}}{|v|_{1, p, K_{\alpha}}^{p}}=\frac{|\hat{v}|_{0, p, \widehat{K}}^{p}}{\left|\hat{v}_{x}\right|_{0, p, \widehat{K}}^{p}+\frac{1}{\alpha^{p}}\left|\hat{v}_{y}\right|_{0, p, \widehat{K}}^{p}} \leq \frac{|\hat{v}|_{0, p, \widehat{K}}^{p}}{\left|\hat{v}_{x}\right|_{0, p, \widehat{K}}^{p}+\left|\hat{v}_{y}\right|_{0, p, \widehat{K}}^{p}}=\frac{|\hat{v}|_{0, p, \widehat{K}}^{p}}{|\hat{v}|_{1, p, \widehat{K}}^{p}} .
$$

This inequality yields

$$
\begin{gathered}
B_{p}\left(K_{\alpha}\right)=\sup _{v \in \Phi_{p}\left(K_{\alpha}\right)} \frac{|v|_{0, p, K_{\alpha}}}{|v|_{1, p, K_{\alpha}}} \leq \sup _{\hat{v} \in \Phi_{p}(\widehat{K})} \frac{|\hat{v}|_{0, p, \widehat{K}}^{p}}{|\hat{v}|_{1, p, \widehat{K}}^{p}}=B_{p}(\widehat{K})<\infty, \\
C_{p}\left(K_{\alpha}\right)=\sup _{v \in \mathcal{E}_{p}\left(K_{\alpha}\right)} \frac{|v|_{0, p, K_{\alpha}}}{|v|_{1, p, K_{\alpha}}} \leq \sup _{\hat{v} \in \mathcal{E}_{p}(\widehat{K})} \frac{|\hat{v}|_{0, p, \widehat{K}}}{|\hat{v}|_{1, p, \widehat{K}}} \leq \sup _{\hat{v} \in \Xi_{p}^{(1,0)}} \frac{|\hat{v}|_{0, p, \widehat{K}}^{p}}{|\hat{v}|_{1, p, \widehat{K}}^{p}}=A_{p}<\infty .
\end{gathered}
$$

Recall that $\widetilde{K}_{\alpha}$ defined in Section 2.4 and depicted in Figure 3 is the triangle with vertices $(0,0)^{\top},(0,1)^{\top},(\alpha s, \alpha t)^{\top}$, where $0<\alpha \leq 1, s^{2}+t^{2}=1$, and $t>0$. Using the inequalities in Section 2.4, we find that

$$
\frac{|v|_{0, p, \widetilde{K}_{\alpha}}}{|v|_{1, p, \widetilde{K}_{\alpha}}} \leq \frac{2^{\eta(p)} t|\hat{v}|_{0, p, K_{\alpha}}}{(1-|s|)^{1 / 2}|\hat{v}|_{1, p, K_{\alpha}}} \leq 2 \frac{|\hat{v}|_{0, p, K_{\alpha}}}{|\hat{v}|_{1, p, K_{\alpha}}}, \quad \forall v \in W^{1, p}\left(\widetilde{K}_{\alpha}\right),
$$

because

$$
2^{\eta(p)}(1+|s|)^{1 / 2} \leq\left\{\begin{array}{ll}
2^{1 / p}, & 1 \leq p \leq 2 \\
2^{1-1 / p}, & 2 \leq p<\infty
\end{array} .\right.
$$

This estimation (4.3) with (4.1) and (4.2) yields

$$
\begin{aligned}
& B_{p}\left(\widetilde{K}_{\alpha}\right):=\sup _{v \in \Phi_{p}\left(\widetilde{K}_{\alpha}\right)} \frac{|v|_{0, p, \widetilde{K}_{\alpha}}}{|v|_{1, p, \widetilde{K}_{\alpha}}} \leq 2 \sup _{\hat{v} \in \Phi_{p}\left(K_{\alpha}\right)} \frac{|\hat{v}|_{0, p, K_{\alpha}}}{|\hat{v}|_{1, p, K_{\alpha}}} \leq 2 B_{p}(\widehat{K}), \\
& C_{p}\left(\widetilde{K}_{\alpha}\right):=\sup _{v \in \mathcal{E}_{p}\left(\widetilde{K}_{\alpha}\right)} \frac{|v|_{0, p, \widetilde{K}_{\alpha}}}{|v|_{1, p, \widetilde{K}_{\alpha}}} \leq 2 \sup _{\hat{v} \in \mathcal{E}_{p}\left(K_{\alpha}\right)} \frac{|\hat{v}|_{0, p, K_{\alpha}}}{|\hat{v}|_{1, p, K_{\alpha}}} \leq 2 A_{p} .
\end{aligned}
$$

The above estimations can be extended to general triangles. Now, let $K$ be an arbitrary triangle. The similar transformation $G_{\beta}: \mathbb{R}^{2} \rightarrow \mathbb{R}^{2}$ for a positive $\beta \in \mathbb{R}$ is defined by $G_{\beta}(\mathbf{x}):=\beta \mathbf{x}$. Let $K_{1}$ be defined by $K_{1}=G_{\beta}(K)$. A function $u \in W^{k, p}(K)$ on $K$ is pulled-back to $v(\mathbf{x}):=u\left(G_{\beta}^{-1}(\mathbf{x})\right)=u\left(G_{1 / \beta}(\mathbf{x})\right)$ on $K_{1}$. Then, for a nonnegative integer $k$ and any $p(1 \leq p \leq \infty)$, we have

$$
|v|_{k, p, K_{1}}=\beta^{2 / p-k}|u|_{k, p, K}, \quad \forall u \in W^{p, k}(K) .
$$


Let $h_{K} \geq h_{1} \geq h_{2}$ be the lengths of the three edges of $K$. Suppose that the second longest edge of $K$ is parallel to the $x$ - or $y$-axis. Then, by a combination of translation, mirror imaging, and $G_{1 / h_{1}}, K$ can be transformed to the triangle $\widetilde{K}_{\alpha}$. Hence, we may apply the above estimations to $\widetilde{K}$ to obtain

$$
\begin{gathered}
\sup _{u \in \Phi_{p}(K)} \frac{|u|_{0, p, K}}{h_{1}|u|_{1, p, K}}=\sup _{v \in \Phi_{p}\left(\widetilde{K}_{\alpha}\right)} \frac{|v|_{0, p, \widetilde{K}_{\alpha}}}{|v|_{1, p, \widetilde{K}_{\alpha}}} \leq 2 B_{p}(\widehat{K}), \\
\sup _{u \in \mathcal{E}_{p}(K)} \frac{|u|_{0, p, K}}{h_{1}|u|_{1, p, K}}=\sup _{v \in \mathcal{E}_{p}\left(\widetilde{K}_{\alpha}\right)} \frac{|v|_{0, p, \widetilde{K}_{\alpha}}}{|v|_{1, p, \widetilde{K}_{\alpha}}} \leq 2 A_{p}
\end{gathered}
$$

and

$$
\sup _{u \in \Phi_{p}(K)} \frac{|u|_{0, p, K}}{|u|_{1, p, K}} \leq 2 B_{p}(\widehat{K}) h_{K}, \quad \sup _{u \in \mathcal{E}_{p}(K)} \frac{|u|_{0, p, K}}{|u|_{1, p, K}} \leq 2 A_{p} h_{K} .
$$

Note that if $p \neq 2$, the Sobolev norms are affected by a rotation. Therefore, we have obtained the following theorem.

Theorem 4.3 Let $K$ be an arbitrary triangle and $h_{K}:=\operatorname{diam} K$. There exists a constant $C=C(p)$ depending only on $p$ such that

$$
\sup _{u \in \Phi_{p}(K)} \frac{|u|_{0, p, K}}{|u|_{1, p, K}} \leq C h_{K}, \quad \sup _{u \in \mathcal{E}_{p}(K)} \frac{|u|_{0, p, K}}{|u|_{1, p, K}} \leq C h_{K}, \quad 1 \leq p \leq \infty .
$$

An important point in Theorem 4.3 is that the constant $C$ is independent of the geometry of $K$.

For $f \in L^{1}(K)$, we define $\bar{f} \in \mathbb{R}$ by

$$
\bar{f}:=\frac{1}{|K|} \int_{K} f(\mathbf{x}) \mathrm{d} \mathbf{x}
$$

From this definition, it is clear that, for arbitrary $f \in L^{p}(K)$,

$$
\int_{K}(f-\bar{f}) \mathrm{d} \mathbf{x}=0 \quad \text { and } \quad|\bar{f}|_{0, p, K} \leq|f|_{0, p, K} .
$$

Hence, we may apply Theorem 4.3 to obtain the Poincaré-Wirtinger inequality for triangles.

Corollary 4.4 (Poincaré-Wirtinger inequality) Let $K$ be an arbitrary triangle. Then, for $p, 1 \leq p \leq \infty$ and the constant $C=C(p)$ that appeared in Theorem 4.3 the following estimation holds:

$$
|f-\bar{f}|_{0, p, K} \leq C h_{K}|f|_{1, p, K}, \quad \forall f \in W^{1, p}(K) .
$$


Remark: The Poincaré-Wirtinger inequality is standard and mentioned in many textbooks. However, the inequality is generally shown under conditions on the domains. For example, it is stated in [3] with the condition that the domain is of $C^{1}$ class. In [8], the inequality (7.45) on page 164 can be read as

$$
|f-\bar{f}|_{0, p, \Omega} \leq\left(\frac{\omega_{d}}{|\Omega|}\right)^{1-1 / d}(\operatorname{diam} \Omega)^{d}|f|_{1, p, \Omega}, \quad \forall f \in W^{1, p}(\Omega),
$$

where $\Omega \subset \mathbb{R}^{d}$ is a bounded convex domain and $\omega_{d}$ is the $(d-1)$-dimensional Hausdorff measure of the unit sphere $S^{d-1} \subset \mathbb{R}^{d}$. Note that if $\Omega$ becomes very "flat", then the coefficient on the right-hand side diverges. For cases of degenerate ("flat") domains, Payne-Weinberger [14] and Laugesen-Siudeja [12] gave estimations for the case $p=2$. Thus, Corollary 4.4 is an extension of prior results.

Because of (4.4), the following lemma obviously holds.

Lemma 4.5 For any $f \in L^{p}(K), 1 \leq p \leq \infty$, we have

$$
|f-\bar{f}|_{0, p, K} \leq 2|f|_{0, p, K} .
$$

We now consider error estimates of the Crouzeix-Raviart interpolation $\mathcal{I}_{K}^{C R} v$. Let $K$ be an arbitrary triangle and $1 \leq p \leq \infty$. From the definition of $\mathcal{I}_{K}^{C R} v$ and the divergence theorem, we notice that

$$
\int_{K}\left(v-\mathcal{I}_{K}^{C R} v\right)_{x} \mathrm{~d} \mathbf{x}=\int_{\partial K}\left(v-\mathcal{I}_{K}^{C R} v\right) n_{1} \mathrm{~d} s=\sum_{i=1}^{3} n_{1} \int_{e_{i}}\left(v-\mathcal{I}_{K}^{C R} v\right) \mathrm{d} s=0
$$

where $\mathbf{n}=\left(n_{1}, n_{2}\right)^{\top}$ is the outer unit normal vector on $\partial K$, which is a constant vector on each edge. Similarly, we have

$$
\int_{K}\left(v-I_{K}^{C R} v\right)_{y} \mathrm{~d} \mathbf{x}=0
$$

Because $\mathcal{I}_{K}^{C R} v \in \mathcal{P}_{1}$ and $\left(\mathcal{I}_{K}^{C R} v\right)_{x},\left(\mathcal{I}_{K}^{C R} v\right)_{y}$ are constants on $K$, these equalities imply that

$$
\begin{aligned}
\left(\mathcal{I}_{K}^{C R} v\right)_{x}= & \frac{1}{|K|} \int_{K} v_{x} \mathrm{~d} \mathbf{x}=: \overline{v_{x}}, \quad\left(\mathcal{I}_{K}^{C R} v\right)_{y}=\frac{1}{|K|} \int_{K} v_{y} \mathrm{~d} \mathbf{x}=: \overline{v_{y}}, \\
& \mathcal{I}_{K}^{C R} v(x, y)=\left(\overline{v_{x}}\right) x+\left(\overline{v_{y}}\right) y+c, \quad c \in \mathbb{R} .
\end{aligned}
$$

Therefore, (4.4) and Poincaré-Wirtinger inequality yield, for arbitrary $v \in W^{2, p}(K)$,

$$
\left|v-\mathcal{I}_{K}^{C R} v\right|_{1, p, K} \leq C h_{K}|v|_{2, p, K} \quad \text { and } \quad\left|\mathcal{I}_{K}^{C R} v\right|_{1, p, K} \leq|v|_{1, p, K} .
$$

Note that $v-\mathcal{I}_{K}^{C R} v \in \mathcal{E}_{p}(K)$ for any $v \in W^{1, p}(K)$. Thus, Theorem 4.3 and 4.5 imply that

$$
\left|v-\mathcal{I}_{K}^{C R} v\right|_{0, p, K} \leq C h_{K}\left|v-\mathcal{I}_{K}^{C R} v\right|_{1, p, K} \leq C^{2} h_{K}^{2}|v|_{2, p, K} \quad \forall v \in W^{2, p}(K) .
$$


Moreover, it follows from Lemma 4.5 that, for $1 \leq p<\infty$,

$$
\begin{aligned}
\left|v-\mathcal{I}_{K}^{C R} v\right|_{1, p, K}^{p} & =\left|v_{x}-\left(\mathcal{I}_{K}^{C R} v\right)_{x}\right|_{0, p, K}^{p}+\left|v_{y}-\left(\mathcal{I}_{K}^{C R} v\right)_{y}\right|_{0, p, K}^{p} \\
& =\left|v_{x}-\overline{v_{x}}\right|_{0, p, K}^{p}+\left|v_{y}-\overline{v_{y}}\right|_{0, p, K}^{p} \\
& \leq 2^{p}\left(\left|v_{x}\right|_{0, p, K}^{p}+\left|v_{y}\right|_{0, p, K}^{p}\right)=2^{p}|v|_{1, p, K}^{p} .
\end{aligned}
$$

The case of $p=\infty$ is similar. Hence, we obtain

$$
\left|v-\mathcal{I}_{K}^{C R} v\right|_{0, p, K} \leq 2 C h_{K}|v|_{1, p, K}, \quad \forall v \in W^{1, p}(K) .
$$

Gathering estimates (4.6) and 4.7), we obtain the following theorem.

Theorem 4.6 Let $K$ be an arbitrary triangle and $1 \leq p \leq \infty$. Then, for the CrouzeixRaviart interpolation $\mathcal{I}_{K}^{C R} v$, the following error estimations hold:

$$
\begin{gathered}
\left|v-\mathcal{I}_{K}^{C R} v\right|_{0, p, K} \leq 2 C h_{K}|v|_{1, p, K}, \quad \forall v \in W^{1, p}(K), \\
\left|v-\mathcal{I}_{K}^{C R} v\right|_{0, p, K} \leq C h_{K}\left|v-\mathcal{I}_{K}^{C R} v\right|_{1, p, K} \leq C^{2} h_{K}^{2}|v|_{2, p, K}, \quad \forall v \in W^{2, p}(K) .
\end{gathered}
$$

Here, the constant $C=C(p)$ is from Theorem 4.3 and is independent of the geometry of $K$.

\section{Approximating the surface area by Crouzeix-Raviart interpolation}

Recall that $\Omega \subset \mathbb{R}^{2}$ is a polygonal domain and $\left\{\tau_{k}\right\}_{k=1}^{\infty}$ is a sequence of triangulations of $\Omega$ with $\lim _{k \rightarrow \infty}\left|\tau_{k}\right|=0$. Let $f \in W^{1, \infty}(\Omega)$. The surface area $A_{L}(f)$ in the sense of Lebesgue is approximated by Crouzeix-Raviart interpolation as

$$
A_{\tau_{k}}^{C R}(f):=\sum_{K \in \tau_{k}} \int_{K} \sqrt{1+\left|\nabla\left(\mathcal{I}_{K}^{C R} f\right)\right|^{2}} \mathrm{~d} \mathbf{x} .
$$

Let $\varepsilon>0$ be arbitrarily taken and fixed. We may take $f_{\varepsilon} \in W^{2,1}(\Omega)$ such that $\left|f-f_{\varepsilon}\right|_{1,1, \Omega}<\varepsilon$. There exists an integer $N$ such that, for any integer $k \geq N$, we have $C\left|\tau_{k}\right|\left|f_{\varepsilon}\right|_{2,1, \Omega}<\varepsilon$, where the constant $C$ is from Theorem 4.6. It follows from (3.2) and 4.5) that, for $k \geq N$,

$$
\begin{aligned}
\mid A_{L}(f)- & A_{\tau_{k}}^{C R}(f)\left|\leq \sum_{K \in \tau_{k}}\right| f-\left.\mathcal{I}_{K}^{C R} f\right|_{1,1, K} \\
& \leq \sum_{K \in \tau_{k}}\left(\left|f-f_{\varepsilon}\right|_{1,1, K}+\left|f_{\varepsilon}-\mathcal{I}_{K}^{C R} f_{\varepsilon}\right|_{1,1, K}+\left|\mathcal{I}_{K}^{C R}\left(f_{\varepsilon}-f\right)\right|_{1,1, K}\right) \\
& \leq 2\left|f-f_{\varepsilon}\right|_{1,1, \Omega}+C\left|\tau_{k}\right|\left|f_{\varepsilon}\right|_{2,1, \Omega}<3 \varepsilon
\end{aligned}
$$

Therefore, we have shown the following theorem.

Theorem 5.1 Let $\Omega \subset \mathbb{R}^{2}$ be a bounded polygonal domain and $\left\{\tau_{k}\right\}_{k=1}^{\infty}$ be a sequence of triangulations of $\Omega$ such that $\lim _{k \rightarrow \infty}\left|\tau_{k}\right|=0$. Let $f \in W^{1, \infty}(\Omega)$ and $A_{\tau_{k}}^{C R}(f)$ be the approximation of the surface area $A_{L}(f)$ by Crouzeix-Raviart interpolation. Then, we have

$$
\lim _{k \rightarrow \infty} A_{\tau_{k}}^{C R}(f)=A_{L}(f) .
$$


It is clear from the proof that 5.1 holds under the assumptions that $f \in W^{1,1}(\Omega) \cap$ $C^{0}(\bar{\Omega})$ with $A_{L}(f)<\infty$ and $f$ is absolutely continuous in the sense of Tonelli. We here strongly emphasize that we have not imposed any geometric conditions on $\left\{\tau_{k}\right\}$, such as the maximum angle condition, or the circumradius condition.

\section{Approximating areas of surfaces in parametric form}

In this section, we show that the results obtained so far can be straightforwardly extended to the case of parametric surfaces. Let $\Omega \subset \mathbb{R}^{2}$ be a bounded polygonal domain and $\mathbf{f}: \Omega \rightarrow \mathbb{R}^{3} \in W^{1, \infty}\left(\Omega ; \mathbb{R}^{3}\right)$. Because the Jacobian matrix

$$
D \mathbf{f}(\mathbf{x}):=\left(\begin{array}{ll}
\frac{\partial f^{1}}{\partial x} & \frac{\partial f^{1}}{\partial y} \\
\frac{\partial f^{2}}{\partial x} & \frac{\partial f^{2}}{\partial y} \\
\frac{\partial f^{3}}{\partial x} & \frac{\partial f^{3}}{\partial y}
\end{array}\right)
$$

exists almost everywhere in $\Omega$, we may assume that $\operatorname{rank} D f(\mathbf{x})=2$ almost everywhere in $\Omega$. Then, the image of $\mathbf{f}$ is a surface (possibly with self-intersections) in $\mathbb{R}^{3}$. Its area $A_{L}(\mathbf{f})$ in the sense of Lebesgue is defined as before (see [19] for details), and is equal to

$$
A_{L}(\mathbf{f})=\int_{\Omega}\left|\mathbf{f}_{x} \times \mathbf{f}_{y}\right| \mathrm{d} \mathbf{x}
$$

where

$$
\mathbf{f}_{x}:=\left(\frac{\partial f^{1}}{\partial x}, \frac{\partial f^{2}}{\partial x}, \frac{\partial f^{3}}{\partial x}\right)^{\top}, \quad \mathbf{f}_{y}:=\left(\frac{\partial f^{1}}{\partial y}, \frac{\partial f^{2}}{\partial y}, \frac{\partial f^{3}}{\partial y}\right)^{\top}
$$

and $\mathbf{f}_{x} \times \mathbf{f}_{y}$ is the exterior product of $\mathbf{f}_{x}, \mathbf{f}_{y}$. The surface area $A_{L}(\mathbf{f})$ can be discussed in terms of the Hausdorff measure and the area formula. See [7, Chapter 4] for details.

We now consider interpolations of $\mathbf{f}$. Let $\left\{\tau_{n}\right\}_{n=1}^{\infty}$ be a sequence of triangulations of $\Omega$. On each $\tau_{n}$, the Lagrange and Crouzeix-Raviart interpolations $\mathcal{I}_{K}^{L} \mathbf{f}, \mathcal{I}_{K}^{C R} \mathbf{f}$ are defined component-wise. Then, $A_{L}(\mathbf{f})$ is approximated by $A_{L}\left(\mathcal{I}_{\tau_{n}}^{L} \mathbf{f}\right)$ and

$$
A_{\tau_{n}}^{C R}(\mathbf{f}):=\sum_{K \in \tau_{n}} \int_{K}\left|\left(\mathcal{I}_{K}^{C R} \mathbf{f}\right)_{x} \times\left(\mathcal{I}_{K}^{C R} \mathbf{f}\right)_{y}\right| \mathrm{d} \mathbf{x},
$$

respectively.

To simplify the notation, we introduce the vectors $\mathbf{F}=\left(F_{1}, F_{2}, F_{3}\right)^{\top}$ and $\mathbf{G}=$ $\left(G_{1}, G_{2}, G_{3}\right)^{\top}$ defined by

$$
\mathbf{F}:=\mathbf{f}_{x} \times \mathbf{f}_{y}, \quad \mathbf{G}:=\mathbf{g}_{x} \times \mathbf{g}_{y},
$$


where $\mathbf{g}:=\mathcal{I}_{K}^{L} \mathbf{f}$ or $\mathbf{g}:=\mathcal{I}_{K}^{C R} \mathbf{f}$. Then, the error $\left|A_{L}(\mathbf{f})-A_{L}\left(\mathcal{I}_{\tau_{n}}^{L} \mathbf{f}\right)\right|$ is estimated as

$$
\begin{aligned}
\left|A_{L}(\mathbf{f})-A_{L}\left(\mathcal{I}_{\tau_{n}}^{L} \mathbf{f}\right)\right| & =\left|\int_{\Omega}\right| \mathbf{F}\left|\mathrm{d} \mathbf{x}-\int_{\Omega}\right| \mathbf{G}|\mathrm{d} \mathbf{x}| \\
& \leq \sum_{i=1}^{3} \int_{\Omega} \frac{\left|F_{i}+G_{i}\right|\left|F_{i}-G_{i}\right|}{|\mathbf{F}|+|\mathbf{G}|} \mathrm{d} \mathbf{x} \\
& \leq \sum_{K \in \tau_{n}} \sum_{i=1}^{3} \int_{K}\left|F_{i}-G_{i}\right| \mathrm{d} \mathbf{x},
\end{aligned}
$$

because $\left|F_{i}+G_{i}\right| /(|\mathbf{F}|+|\mathbf{G}|) \leq 1$. The error $\left|A_{L}(\mathbf{f})-A_{\tau_{n}}^{C R}(\mathbf{f})\right|$ is estimated in a similar manner.

Note that $F_{i}$ and $G_{i}$ are written as

$$
F_{i}=f_{x}^{k} f_{y}^{l}-f_{y}^{k} f_{x}^{l}, \quad G_{i}=g_{x}^{k} g_{y}^{l}-g_{y}^{k} g_{x}^{l}, \quad k, l=1,2,3, k \neq l,
$$

where $g^{i}=\mathcal{I}_{K}^{L} f^{i}$ or $g^{i}=\mathcal{I}_{K}^{C R} f^{i}$. Therefore, we see that

$$
\left|F_{i}-G_{i}\right| \leq\left|f_{x}^{k}-g_{x}^{k}\right|\left|f_{y}^{l}\right|+\left|g_{x}^{k}\right|\left|f_{y}^{l}-g_{y}^{l}\right|+\left|f_{y}^{k}-g_{y}^{k}\right|\left|f_{x}^{l}\right|+\left|g_{y}^{k}\right|\left|f_{x}^{l}-g_{x}^{l}\right|
$$

and

$$
\sum_{i=1}^{3} \int_{K}\left|F_{i}-G_{i}\right| \mathrm{d} \mathbf{x} \leq\left(|\mathbf{f}|_{1, \infty, K}+|\mathbf{g}|_{1, \infty, K}\right)|\mathbf{f}-\mathbf{g}|_{1,1, K}
$$

For the case of Lagrange interpolation, $\mathbf{g}=\mathcal{I}_{K}^{L} \mathbf{f}$ and we assume that a sequence of triangulations $\left\{\tau_{n}\right\}$ of $\Omega$ satisfies the maximum angle condition. Then, by Lemma 3.1. there exists a constant $C_{1}$ such that $\left|\mathcal{I}_{K}^{L} \mathbf{f}\right|_{1, \infty, K} \leq C_{1}|\mathbf{f}|_{1, \infty, K}$, where the constant $C_{1}$ depends on the maximum angle. Thus, we have

$$
\begin{aligned}
\left|A_{L}(\mathbf{f})-A_{L}\left(\mathcal{I}_{\tau_{n}}^{L} \mathbf{f}\right)\right| & =\left(1+C_{1}\right) \sum_{K \in \tau_{n}}|\mathbf{f}|_{1, \infty, K}|\mathbf{f}-\mathbf{g}|_{1,1, K} \\
& \leq\left(1+C_{1}\right)|\mathbf{f}|_{1, \infty, \Omega}\left|\mathbf{f}-\mathcal{I}_{\tau_{n}}^{L} \mathbf{f}\right|_{1,1, \Omega} .
\end{aligned}
$$

Similarly, for the case of Crouzeix-Raviart interpolation, we have $\mathbf{g}=\mathcal{I}_{K}^{C R} \mathbf{f}$ and

$$
\left|A_{L}(\mathbf{f})-A_{\tau}^{C R}(\mathbf{f})\right| \leq 2 \sum_{K \in \tau_{n}}|\mathbf{f}|_{1, \infty, K}\left|\mathbf{f}-\mathcal{I}_{K}^{C R} \mathbf{f}\right|_{1,1, K}
$$

without any geometric condition on the triangulations.

From these inequalities, the following theorem can be shown in exactly the same manner as used in Sections 3 and 5.

Theorem 6.1 Let $\Omega \subset \mathbb{R}^{2}$ be a bounded polygonal domain and $\left\{\tau_{k}\right\}$ be a sequence of triangulations of $\Omega$. Let $\mathbf{f}: \Omega \rightarrow \mathbb{R}^{3}$ belong to $W^{1, \infty}\left(\Omega ; \mathbb{R}^{3}\right)$ and $\operatorname{rank} D \mathbf{f}(\mathbf{x})=2$ almost everywhere in $\Omega$.

If $\left\{\tau_{k}\right\}$ satisfies the maximum angle condition, we have the convergence

$$
\lim _{k \rightarrow \infty} A_{L}\left(\mathcal{I}_{\tau_{k}}^{L} \mathbf{f}\right)=A_{L}(\mathbf{f})
$$


for Lagrange interpolation. Furthermore, we have

$$
\lim _{k \rightarrow \infty} A_{\tau_{k}}^{C R}(\mathbf{f})=A_{L}(\mathbf{f})
$$

for Crouzeix-Raviart interpolation without any geometric condition on the triangulation $\left\{\tau_{k}\right\}$.

\section{Numerical experiments and concluding remarks}

To confirm the results obtained in this paper, we conducted numerical experiments. Let $\Omega:=(-1,1) \times(-1,1)$ and $N$ be a positive integer. We use the triangulation $\tau$ that consists of congruent isosceles triangles with base length $h:=2 / N$ and height $2 /\left\lfloor 2 / h^{\alpha}\right\rfloor \approx h^{\alpha}, \alpha>1$. Note that the circumradius of the triangle is approximately equal to $h^{\alpha} / 2+h^{2-\alpha} / 8$. Thus, it diverges when $\alpha>2$ as $N \rightarrow \infty$. The triangulation of $\Omega$ with $N=12$ and $\alpha=1.6$ is shown in Figure 4 .

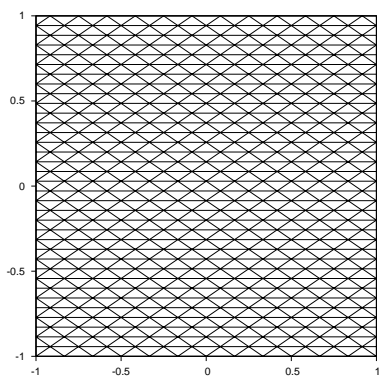

Fig. 4 The triangulation of $\Omega$ with $N=12$ and $\alpha=1.6$.

Let $f(x, y):=\left(a^{2}-x^{2}\right)^{1 / 2}$ with $a=1.1$. We computed $\left|A_{L}(f)-A_{E}\left(\mathcal{I}_{\tau}^{L} f\right)\right|$ and $\left|A_{L}(f)-A_{\tau}^{C R}(f)\right|$ with various $N$ and $\alpha$. The results are shown in Figure 5.

Note that, as predicted by the error estimations obtained in this paper, the behavior of the error $\left|A_{L}(f)-A_{\tau}^{C R}(f)\right|$ does not depend on $\alpha$ (all the curves overlap and look like just one curve), whereas the error $\left|A_{L}(f)-A_{E}\left(\mathcal{I}_{\tau}^{L} f\right)\right|$ behaves differently as $\alpha$ varies. We can also see that, when $N$ is small, the errors in the Lagrange interpolation behave strangely for some reason that the authors cannot explain.

We obtained an alternative proof of the classical result by Young (Theorem 3.2). That is, we have shown that the areas of the Lagrange interpolation of a surface (of class $W^{1, \infty}$ ) converge to the area of the surface under the maximum angle condition on the triangulation. The authors conjecture that the same result holds under the circumradius condition. Moreover, we showed that the areas of the Crouzeix-Raviart interpolation of a surface (of class $W^{1, \infty}$ ) converge to the area of the surface without any geometric condition on the triangulation.

The authors believe that the results of this paper provide a new insight on the definition of surface area and related subjects. In the following, we mention some immediate problems that arise from this study.

- Prove or disprove the conjecture that Theorem 3.2 holds under the circumradius condition on triangulations. 

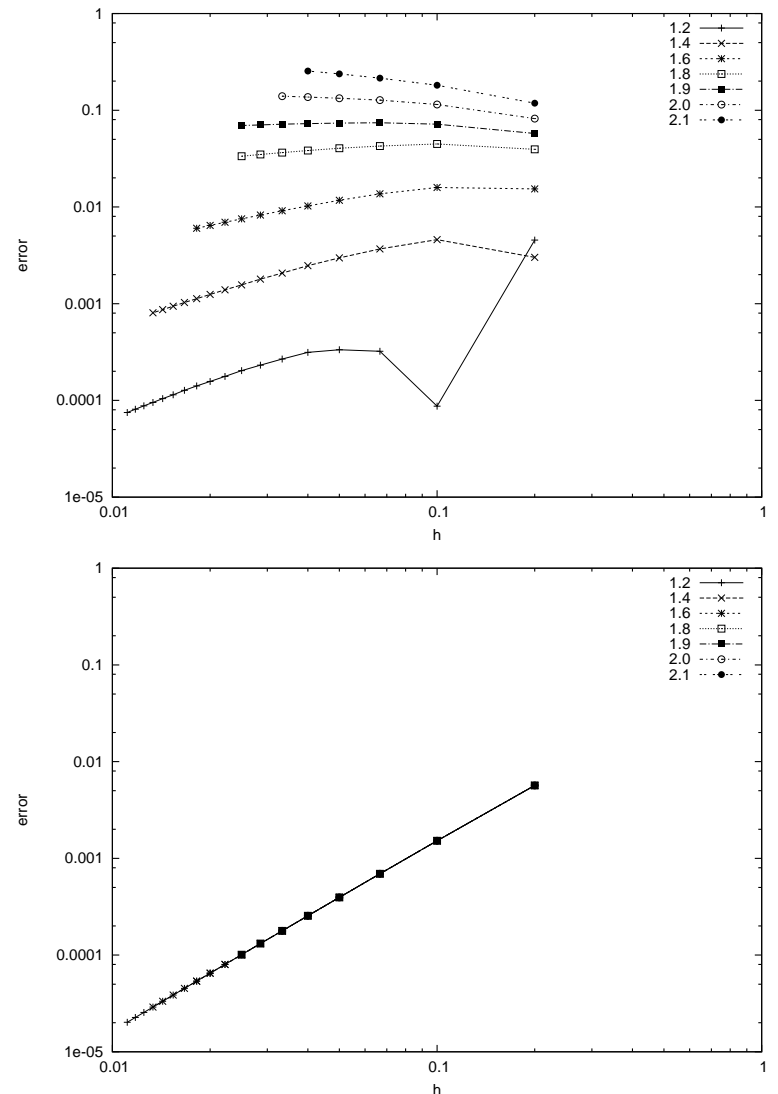

Fig. 5 The errors of Lagrange (upper) and Crouzeix-Raviart (lower) interpolations. The number next to the symbol indicates the value of $\alpha$. The horizontal axis represents the maximum size of triangles and the vertical axis represents the errors $\left|A_{E}\left(\mathcal{I}_{\tau_{k}}^{L} f\right)-A_{L}(f)\right|$ (upper) and $\left|A_{\tau_{k}}^{C R}(f)-A_{L}(f)\right|$ (lower).

- The surface area in the sense of Lebesgue is defined using Lagrange interpolation (or using the subspace $S_{\tau_{n}}$ ). Can we give an alternate definition of surface area using Crouzeix-Raviart interpolation (or using a corresponding finite dimensional space) that is equivalent to the original definition?

- All the results in this paper are proved under the assumption $A_{L}(f)<\infty$. Let $f \in C^{0}(\Omega)$ and $\left\{\tau_{k}\right\}$ be a sequence of triangulations such that $\lim _{k \rightarrow \infty}\left|\tau_{k}\right|=0$. In this case, the Crouzeix-Raviart interpolation $\mathcal{I}_{\tau_{k}}^{C R} f$ is well-defined. Suppose that $\limsup _{k \rightarrow \infty} A_{\tau_{k}}^{C R}(f)<\infty$. Then, can we show that $A_{L}(f)<\infty$ ? If not, give a counter-example.

- Extend Theorem 5.1 to the case of the volume of the graph of a function with $d$ variables, $d \geq 3$.

The authors hope this paper will inspire further research and that one or more of the above-mentioned questions will be solved in the near future. 
AcKNOWLEDGEMENTS. The authors were supported by JSPS KAKENHI Grant Numbers JP16H03950, JP25400198, and JP26400201.

\section{References}

1. I. Babuška, A.K. Aziz: On the angle condition in the finite element method, SIAM J. Numer. Anal., 13 (1976), 214-226.

2. S.C. Brenner, L.R. Scott: The Mathematical Theory of Finite Element Methods, 3rd edition, Springer, 2008.

3. H. Brezis: Functional Analysis, Sobolev Spaces, and Partial Differential Equations, Springer, 2011.

4. K. Cesari: Surface Area, Princeton Univ. Press, 1956.

5. P.G. Ciarlet: The Finite Element Methods for Elliptic Problems, North Holland, 1978, reprint by SIAM 2008.

6. A. Ern, J-L. Guermond: Theory and Practice of Finite Elements, Springer, 2004.

7. L.C. Evans, R.F. Gariepy: Measure Theory and Fine Properties of Functions, CRC Press, 1992.

8. D. Gilbarg, N.S. Trudinger: Elliptic Partial Differential Equations of Second Order, 2nd edition, Springer, 1993.

9. K. Kobayashi, T. Tsuchiya: A Babuška-Aziz type proof of the circumradius condition, Japan J. Indust. Appl. Math., 31 (2014), 193-210.

10. K. Kobayashi, T. Tsuchiya: On the circumradius condition for piecewise linear triangular elements, Japan J. Indust. Appl. Math., 32 (2015) 65-76.

11. K. Kobayashi, T. Tsuchiya: A priori error estimates for Lagrange interpolation on triangles. Appl. Math., Praha 60 (2015), 485-499.

12. R.S. Laugesen, B.A. Siudeja: Minimizing Neumann fundamental tones of triangles: An optimal Poincaré inequality, J. Differential Equations, 249 (2010), 118-135.

13. X. Liu, F. Kikuchi: Analysis and estimation of error constants for $P_{0}$ and $P_{1}$ interpolations over triangular finite elements, J. Math. Sci. Univ. Tokyo, 17 (2010), 27-78.

14. L.E. Payne, H.F. Weinberger: An optimal Poincaré inequality for convex domains, Arch. Ration. Mech. Anal., 6 (1960), 286-292.

15. H. Rademacher: Über partielle und totale differenzierbarkeit von funktionen mehrerer vaiabeln und über die transformation der doppelintegrale, Math. Ann., 79, 340-359 (1919) doi 10.1007/BF01498415

16. H. Rademacher: Über partielle und totale differenzierbarkeit von funktionen mehrerer vaiabeln II, Math. Ann., 81, 52-63 (1920) doi 10.1007/BF01563620

17. T. Radò: On the Problem of Plateau, Springer, 1933, reprinted by Chelsea, 1951.

18. T. Radò: Length and Area, American Mathematical Society, 1948.

19. S, Saks: Theory of the Integral, Warszawa-Lwów, 1937, reprint by Dover 2005.

20. W.H. Young: On the triangulation method of defining the area of a surface, Proc. London Math. Soc., 19, 117-152 (1921) doi:10.1112/plms/s2-19.1.117 